\title{
BMJ Open Arm Based on LEg blood pressures (ABLE-BP): can systolic leg blood pressure measurements predict systolic brachial blood pressure? Protocol for an individual participant data meta- analysis from the INTERPRESS-IPD Collaboration
}

\author{
Sinead T J McDonagh (D) ,' James P Sheppard (D) ,2 Fiona C Warren, ${ }^{1}$ \\ Kate Boddy (1) , ${ }^{3}$ Leon Farmer, ${ }^{4}$ Helen Shore, ${ }^{4}$ Phil Williams, ${ }^{4}$ Philip S Lewis, ${ }^{5}$ \\ Rachel Baumber, ${ }^{6}$ Jayne Fordham, ${ }^{7}$ Una Martin, ${ }^{8}$ Victor Aboyans, ${ }^{9}$ \\ Christopher E Clark (D) , ${ }^{1}$ on behalf of the INTERPRESS-IPD Collaborators
}

To cite: McDonagh STJ, Sheppard JP, Warren FC, et al. Arm Based on LEg blood pressures (ABLE-BP): can systolic leg blood pressure measurements predict systolic brachial blood pressure? Protocol for an individual participant data meta-analysis from the INTERPRESS-IPD Collaboration. BMJ Open 2021;11:e040481. doi:10.1136/ bmjopen-2020-040481

- Prepublication history for this paper is available online. To view these files, please visit the journal online (http://dx.doi. org/10.1136/bmjopen-2020040481).

Received 15 May 2020 Revised 13 December 2020 Accepted 09 January 2021

Check for updates

(c) Author(s) (or their employer(s)) 2021. Re-use permitted under CC BY-NC. No commercial re-use. See rights and permissions. Published by BMJ.

For numbered affiliations see end of article.

Correspondence to Dr Christopher E Clark; c.e.clark@exeter.ac.uk

\section{ABSTRACT}

Introduction Blood pressure (BP) is normally measured on the upper arm, and guidelines for the diagnosis and treatment of high BP are based on such measurements. Leg BP measurement can be an alternative when brachial BP measurement is impractical, due to injury or disability. Limited data exist to guide interpretation of leg BP values for hypertension management; study-level systematic review findings suggest that systolic $B P(S B P)$ is $17 \mathrm{~mm}$ $\mathrm{Hg}$ higher in the leg than the arm. However, uncertainty remains about the applicability of this figure in clinical practice due to substantial heterogeneity.

Aims To examine the relationship between arm and leg SBP, develop and validate a multivariable model predicting arm SBP from leg SBP and investigate the prognostic association between leg SBP and cardiovascular disease and mortality.

Methods and analysis Individual participant data (IPD) meta-analyses using arm and leg SBP measurements for 33710 individuals from 14 studies within the Inter-arm blood pressure difference IPD (INTERPRESSIPD) Collaboration. We will explore cross-sectional relationships between arm and leg SBP using hierarchical linear regression with participants nested by study, in multivariable models. Prognostic models will be derived for all-cause and cardiovascular mortality and cardiovascular events.

Ethics and dissemination Data originate from studies with prior ethical approval and consent, and data sharing agreements are in place-no further approvals are required to undertake the secondary analyses proposed in this protocol. Findings will be published in peerreviewed journal articles and presented at conferences. A comprehensive dissemination strategy is in place, integrated with patient and public involvement. PROSPERO registration number CRD42015031227.

\section{Strengths and limitations of this study}

- This individual participant data (IPD) meta-analysis uses the INTERPRESS-IPD Collaboration (IPD from 24 international cohorts, originally created to explore the association between interarm differences in blood pressure (BP) and mortality risk), the largest known dataset to allow an in-depth exploration of the relationship between arm and leg systolic BP (SBP) and the role of leg SBP in cardiovascular risk estimation.

- An IPD approach maximises statistical power and allows a consistent approach toward all available data that cannot be achieved with study-level meta-analyses.

- Inclusion of a number of international cohorts in this IPD meta-analyses will maximise the generalisability of the findings.

- Methods of data collection and reporting of results vary between included cohorts and this is acknowledged as a limitation of the data. We are aware of other studies with arm and leg BP data that are not included in the INTERPRESS-IPD Collaboration. However, the dataset is large enough to allow robust analysis and sufficient subgroup and sensitivity analyses to answer questions that cannot be addressed by study-level meta-analyses

- Patient and public involvement (PPI) activities have been, and will be, undertaken throughout every stage of this project and we include three PPI advisors and a PPI facilitator as coauthors.

\section{INTRODUCTION}

Blood pressure (BP) is normally measured on the upper arm, and all guidelines for the 
diagnosis and treatment of high BP are based on such measurements. ${ }^{1-3}$ When brachial BP measurement is not possible, other measurement sites are required. Uncertainty over interpretation of non-brachial BP measurement may result in inaccurate BP estimates, leading to suboptimal management of hypertension, risking avoidable cerebrovascular or ischaemic cardiac events. ${ }^{4}$ In the clinical setting, this may be a temporary problem due, for example, to fractures, wounds, vascular access devices or during surgical procedures. However, for some people, there are permanent barriers to brachial BP measurement, such as amputation, bilateral lymphoedema (eg, after bilateral mastectomy for breast cancer) or phocomelia (eg, secondary to thalidomide). ${ }^{5}$ Brachial BP measurement may also be inaccurate, and difficult to self-administer, where there is altered muscle tone or hemiplegia following stroke. ${ }^{67}$ It is also unreliable in the presence of bilateral subclavian, axillary or brachial artery stenoses due to atheroma or arteritides. ${ }^{8}$ In any of these circumstances, measurement of BP in the leg is a suitable alternative for monitoring BP, diagnosing and treating hypertension. However, at present, only limited data exist to guide interpretation of the leg systolic $\mathrm{BP}(\mathrm{SBP})$ values.

Historically, ranges of $10-40 \mathrm{~mm} \mathrm{Hg}$ have been suggested for the difference (ie, leg minus arm) between SBP measured in the arm and leg in healthy individuals. ${ }^{910}$ Recently, a systematic review and study level meta-analysis of observational studies were published examining this relationship. ${ }^{11}$ Based on 44 included studies, totalling 9771 participants, ankle SBP was found to be $17.0 \mathrm{~mm}$ $\mathrm{Hg}(95 \%$ CIs 15.4 to $21.3 \mathrm{~mm} \mathrm{Hg}$ ) higher than arm BP in the general population; for diastolic BP, there was no difference. These findings suggested that a threshold of $155 / 90 \mathrm{~mm} \mathrm{Hg}$ in the leg (equating to the National Institute for Health and Care Excellence (NICE) threshold of $140 / 90 \mathrm{~mm} \mathrm{Hg}$ in the arm $)^{3}$ might be used for diagnosing hypertension when ankle BPs are the only measurements available. However, significant statistical heterogeneity was observed in all analyses, which could not be explained in subgroup or sensitivity analyses according to cardiovascular disease history, cardiovascular disease risk, measurement method and device or methodological quality. Metaregression by age and arm SBP level was also uninformative. $^{11}$

Study-level aggregate meta-analyses are limited in the conclusions that can be drawn, because they combine studies with different patient characteristics (eg, age or coexisting disease), methodological choices (eg, posture in BP measurement or sequential vs simultaneous measurement) and analytical approaches. These limitations can potentially be overcome by obtaining the original individual participant data (IPD) from cohorts. ${ }^{12}$ Such IPD meta-analyses, while time consuming, offer advantages, such as checking of modelling assumptions, analysing variables on continuous scales and the possibility of assessing for non-linear relationships. ${ }^{13}$ They offer the ability to uniformly adjust findings for other variables, thus potentially accounting and adjusting for heterogeneity between findings in a way that study-level meta-analyses cannot. ${ }^{14}$

We propose to undertake IPD meta-analyses to answer the following research questions:

1. What is the mean difference, in the absence of peripheral arterial disease, between SBP measured in the arm and SBP measured in the leg in the same individuals?

2. To what extent do these differences vary according to patient characteristics and methods of measurement, and what are the impacts of cerebrovascular and cardiac diseases on the difference between arm and leg pressures?

3. Can a model be developed and validated to predict arm SBP, based on leg SBP measurements and other patient characteristics, to inform interpretation of individual leg SBP readings?

4. How does leg BP, in comparison with models based on arm BP, predict cardiovascular events and/or mortality?

\section{METHODS AND ANALYSIS}

\section{Aims and objectives}

This IPD meta-analysis has the following aims

1. To examine the relationship between arm and leg SBP, taking into account patient characteristics such as age, baseline BP and medical history.

2. To derive and validate a prediction model to permit estimation of an equivalent brachial SBP based on leg SBP measurements.

3. To determine the independent prognostic value of leg SBP in predicting cardiovascular events and mortality risk.

\section{Data sources and description of the dataset}

This study will use an observational cohort design, undertaking IPD meta-analyses of data held by the interarm BP difference (INTERPRESS-IPD) Collaboration, established to undertake IPD meta-analyses examining the independent contribution of interarm BP difference to prediction of mortality and cardiovascular events. ${ }^{15}$ The establishment of the Collaboration has been previously described. ${ }^{15}$ In brief, literature searches and author contacts were used to identify studies likely to hold records of BP in both arms. A subset of these studies measured Ankle-Brachial Index (ABI) at recruitment, thus providing data for arm and leg BPs. ${ }^{16}$ Individual data sharing agreements are in place with the lead authors of each participating study; their consent has been obtained for the proposed analyses and corresponding authors for each participating study will contribute to publications arising from these analyses. Core data, held for the primary INTERPRESS-IPD research outputs, will undergo additional cleaning and merging of relevant additional variables prior to combination into a new, expanded, single dataset.

The new Arm Based on LEg-BP (ABLE-BP) dataset will include 33710 individual records from 14 European, 
USA and African studies that measured both arm and leg BP. Participants in the dataset have a mean age of 58 years (range: 18-99 years), 45\% are women and mean systolic/ diastolic brachial BP is $135 / 80 \mathrm{~mm} \mathrm{Hg}$. In total, 20191 $(60 \%)$ have hypertension (defined as a formal clinical diagnosis and/or on antihypertensive treatment), 4917 $(15 \%)$ have diabetes, $5474(17 \%)$ have pre-existing ischaemic heart disease and $1900(6 \%)$ have had a cerebrovascular event. Median follow-up period is 8.0 years, with 2811 (9\%) participants experiencing cardiovascular events or death and 621 (2\%) dying within 10 years. We will present tables including descriptors (eg, country, method of BP measurement, description of cohort) of each study to assess comparability and describe the dataset. A summary of the included studies and their characteristics is given in table 1 .

\section{Outcomes}

The primary outcome (systolic arm-leg BP difference) for the analyses will be defined as the lower leg posterior tibial artery BP minus the higher arm BP measured on the brachial artery. The coprimary outcome will be arm SBP predicted from leg BP. Primary analyses will use observed data only (see missing data-below).

Secondary outcomes are the prognostic value of leg BPs for prediction of cardiovascular events and mortality.

\section{Quality assessment}

The methodological quality and risk of bias for studies contributing data has been assessed using the Quality assessment In Prognostic Studies (QUIPS) score, modified for IPD analysis. ${ }^{17}$ These assessments will be used to inform sensitivity analyses focusing on the highest quality studies. This quality assessment covers domains on selection bias, attrition, and accuracy of measurement, analysis and confounding.

\section{Participant selection}

Participants with ankle or arm BP missing at recruitment will be excluded from the analyses. We will also exclude participants with a diagnosis of peripheral arterial disease, low ABI $(<0.90)$ and those studies where participant entry criteria was based on selected ABI.

\section{Statistical analysis}

\section{Descriptive analyses}

Descriptive statistics will be used to describe participant characteristics at the study level, including age, sex, ethnic group, body mass index (BMI), arm and leg BP, and history of cardiovascular diseases (and risk factors). Data will be presented as means with $\mathrm{SD}$, median with IQR or proportions.

\section{Investigation of relationship between leg and arm BP}

We will report the mean arm-leg differences for each study. These will be examined in a two-stage meta-analysis. Estimates of heterogeneity from these analyses will be used to determine whether to conduct a further one-stage analysis with study entered as a random or as a fixed effect.
We will explore cross-sectional relationships between arm and leg BP in univariable and multivariable models with all available data, using hierarchical linear regression. Estimates will be adjusted for age, sex, baseline BP, smoking status, serum cholesterol and medical history at recruitment. Recording of medication use varies across cohorts; we will perform secondary analyses that include use of specific classes of antihypertensive medication (eg, calcium channel blockers, renin-angiotensin system blockers) using data from only those studies that recorded the relevant information. Should drug use be a significant predictor of outcome when included with other significant variables, it will be retained in the models derived from these secondary analyses. Depending on the results of our quality assessment of primary studies, we will perform sensitivity analyses to include only those studies evaluated to be at low risk of bias. No further secondary or sensitivity analyses are planned.

\section{Prediction modelling of arm BP using leg BP}

Using a subset of participants with complete case data for candidate variables both identified above, and set a priori, we will model brachial SBP on leg SBP using random effects meta-analysis models. We will use one-stage and two-stage methods, and assess heterogeneity using the $\mathrm{I}^{2}$ and $\mathrm{tau}^{2}$ statistics. One-stage models will comprise hierarchical linear regression models (participants nested by study). Further models will investigate the association between arm-leg difference and participant characteristics (using a series of models with one characteristic per model). Predictor variables to be included a priori in the modelling will include age, sex, BMI, smoking status, ethnicity, diagnosis of diabetes, hypertension or any cardiovascular disease, total cholesterol and baseline ankle BP.

The predictive model for arm SBP will be developed using one-stage meta-analysis with hierarchical linear regression models, as described above. We will derive the model using a subset of the complete case data (derivation dataset) and validate the model using the remaining data (validation dataset). ${ }^{18}$ The primary studies will be allocated to the derivation or validation datasets such that both datasets include participants of both genders and reflect the geographical origin of the studies.

\section{Prognostic modelling}

Prognostic models based on leg SBP will be derived for all-cause and cardiovascular mortality and fatal or nonfatal cardiovascular events. Heterogeneity will be assessed using $\mathrm{I}^{2}$ and $\mathrm{tau}^{2}$. We will aim to perform one-stage random effects time-to-event models based on flexible parametric models; should such models fail to converge, we will use fixed effect Cox proportional hazards models, stratified by study. Using the covariates described above, and again dividing the dataset into a derivation and validation cohort, we will derive and validate a suitable model. For prognostic modelling, we will exclude participants with any pre-existing cardiovascular disease. 


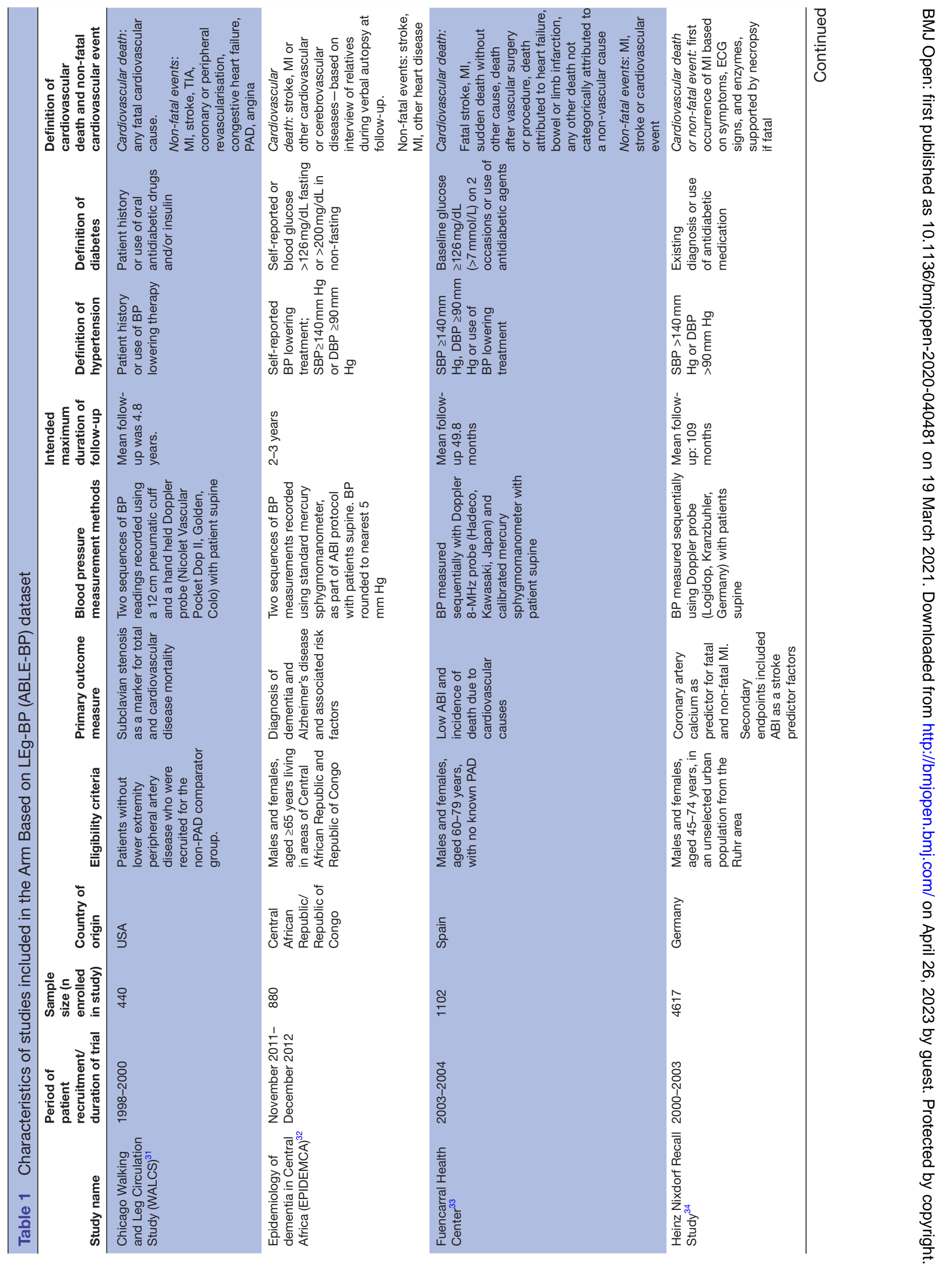




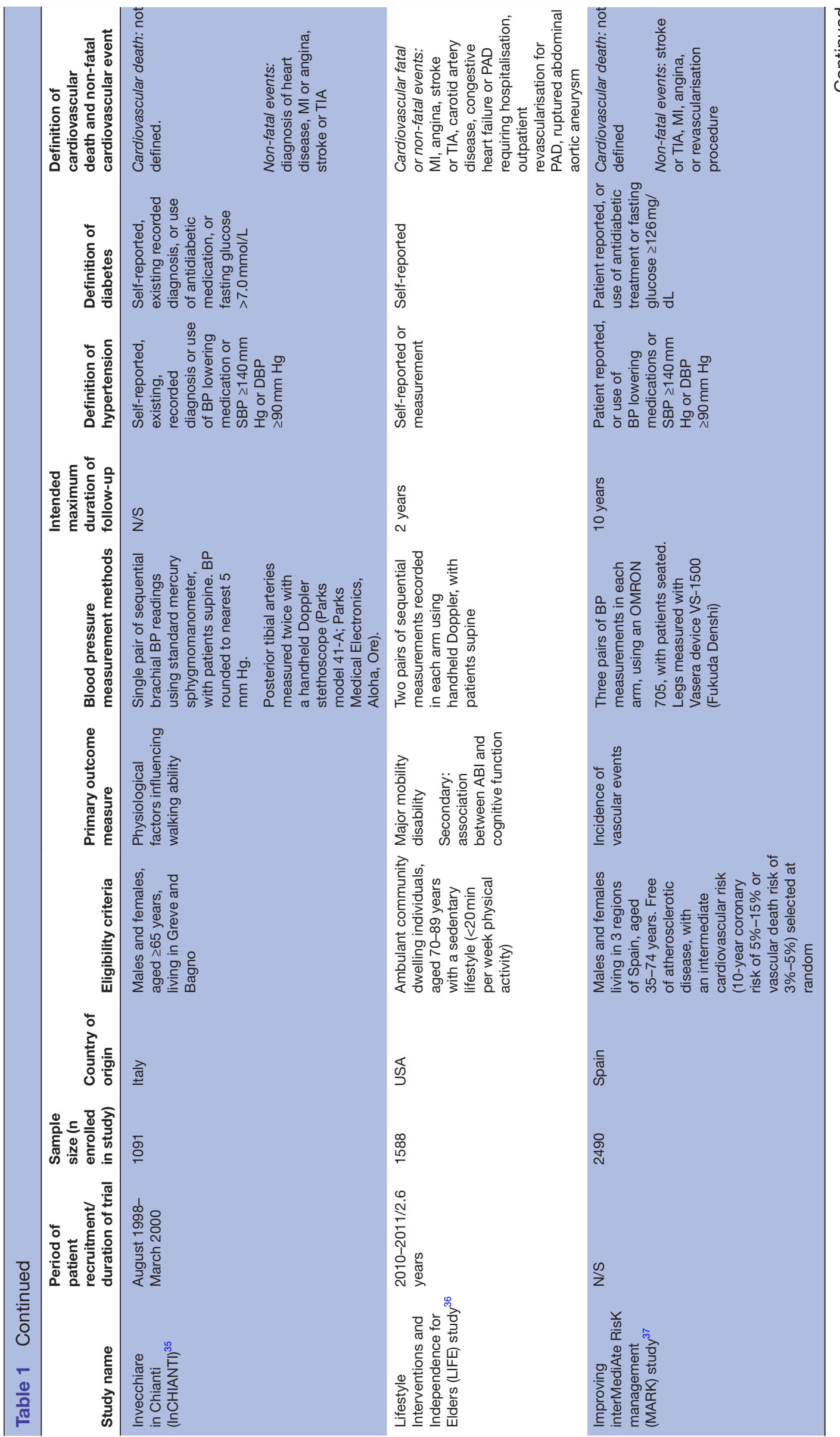




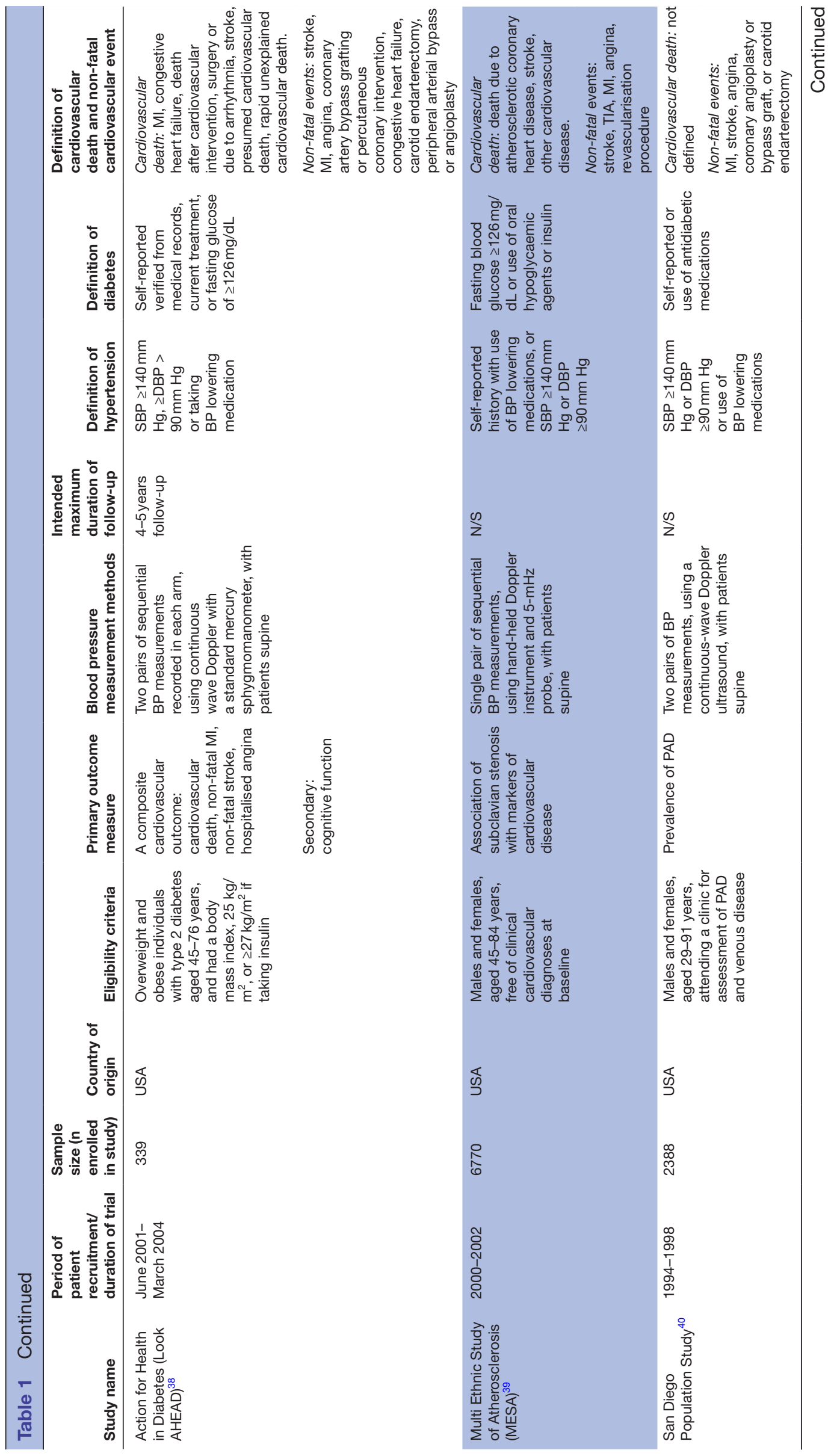




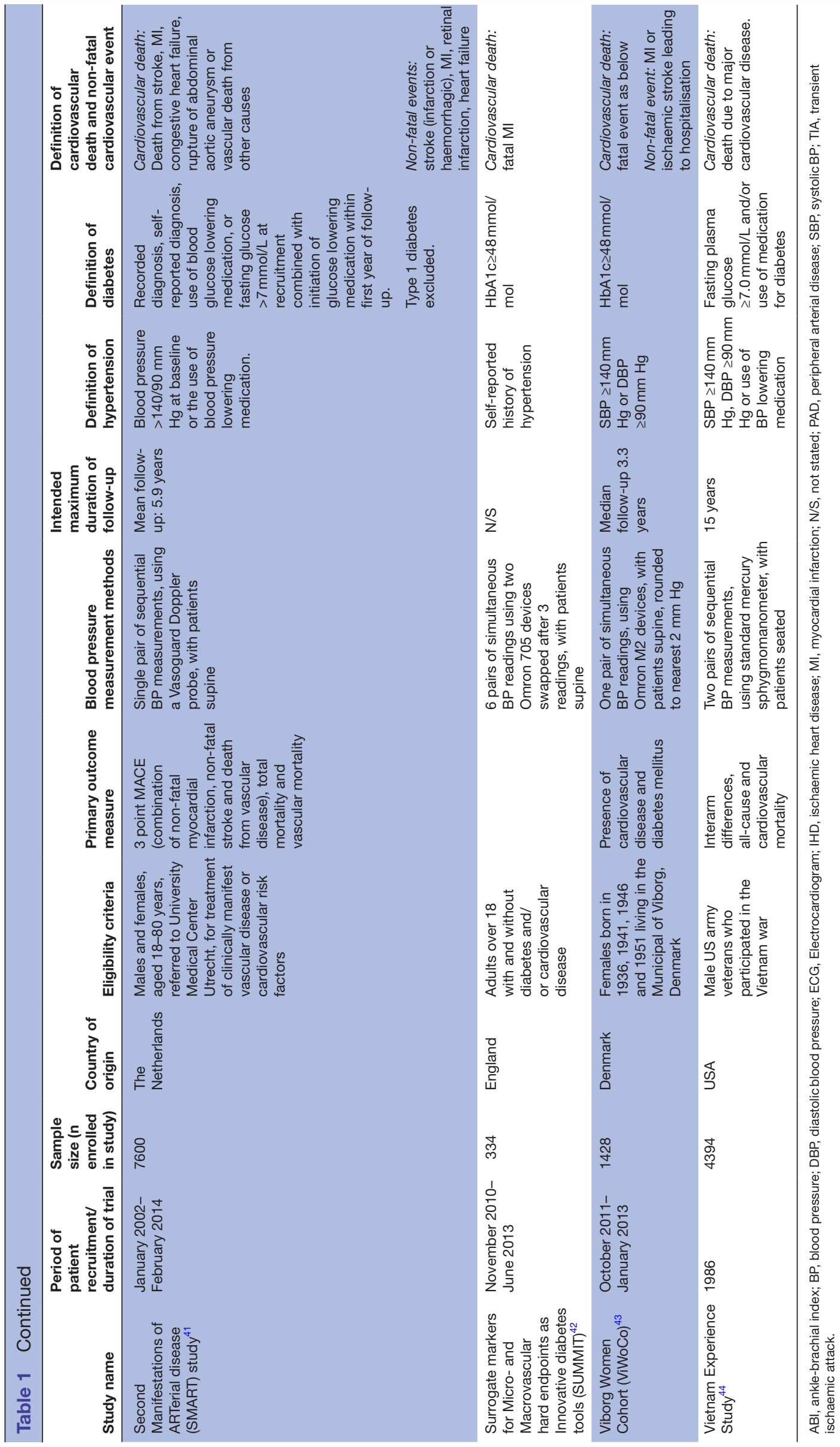


Using internationally recognised 10-year risk scores, such as the European Systematic COronary Risk Evaluation (SCORE) and Atherosclerotic Cardiovascular Disease (ASCVD) pooled cohort equations, we will compare the outcome of such cardiovascular risk scores using arm based on leg SBP data with the actual arm SBP data. ${ }^{19-22}$ Besides their wide use in clinical practice, these two scores have been selected to assess two different outcomes, as SCORE predicts cardiovascular mortality, while ASCVD predicts fatal and non-fatal cardiovascular events (cardiovascular death, non-fatal MI and stroke). Model goodness of fit will be compared using the likelihood ratio test, the Akaike Information Criterion, ${ }^{23}$ and for time-to-event models, the Harrell's C statistic.

\section{Missing data and sensitivity analyses}

For all included studies, the primary analyses will use observed data only. Participants from other cohorts included within the INTERPRESS-IPD Collaboration lack leg BP data but do have brachial BP measurements and ABIs. We will explore whether accurate back-calculation of leg pressures is feasible using these data. To achieve this, we will establish a clear understanding of the study formulae used to derive ABI, including discussion with authors as necessary. We will then trial this approach using datasets that do contain leg pressures to confirm validity. If feasible, we will back-calculate missing leg SBPs and add these data to the observed data for sensitivity analyses to check the primary models. We will also perform sensitivity analyses incorporating height into the final models, where available. Further sensitivity analyses, using multiple imputation of arm and/or leg SBP and participant data for the one-stage meta-analyses where arm-leg or arm SBP is the outcome, and for the timeto-event analyses will also be undertaken. The results of these models will be compared with the primary outcome models using observed data only. Finally, we will repeat the primary analyses excluding studies deemed to be of low or moderate quality based on modified QUIPS scores.

\section{Publication and inclusion bias}

Inclusion bias will be assessed by comparing our pooled estimate of the mean arm-leg SBP difference for studies included in the ABLE-BP analyses with studies using sequential BP measurement methods in our previous study-level systematic review using a two-stage metaanalysis. ${ }^{11}$ Publication bias will not be assessed; we believe that there is limited potential for publication bias, as the primary studies from which we derive data were not originally designed to compare arm and leg BPs. Although we are performing secondary analyses in a subset of an established dataset (INTERPRESS-IPD Collaboration), which is an efficient and cost-effective approach, we must acknowledge that the INTERPRESS-IPD dataset was not established for the purpose of defining the arm-leg SBP relationship and therefore there is a possibility that other data exist that fall outside the scope of the original search terms.

\section{Patient and public involvement}

The development of this protocol has had considerable patient and public involvement (PPI). Prior to funding, a draft was reviewed by three public advisors improving the overall clarity in general, and in specific areas, such as focussing the research questions on aspects of arm and leg BP that interest users. We convened two prefunding PPI workshops to raise awareness about involvement in systematic reviews and gain critical feedback for the project. This feedback resulted in a clearer definition of the population being studied, greater clarity about benefits for patients and reinforcement of our user dissemination plans. We have established a PPI advisory group for the project, led by KB (an academic PPI facilitator) and comprising one stroke survivor and two Thalidomide Trust beneficiaries; they will shape the research by fully participating in quarterly management meetings. The group have contributed towards drafting this protocol and the plain English abstract. We plan two key workshops to ensure that the review findings reach the end user in an accessible way. First, a summary writing workshop with the PPI advisory group to achieve a clear plain language summary and to coproduce a dissemination plan targeted at patients and the public. Second, we will convene a larger public event on the subject of understanding cardiovascular risk, within which the findings of this research can be presented in context.

\section{Ethics and dissemination}

This is a secondary analysis of anonymised IPD which has been obtained from studies where participants have already given consent and approval to participate (see 'ethics approval and patient consent for publication' declaration). We have sought written permission for use of IPD from each individual study lead investigator included in the INTERPRESS-IPD Collaboration. We will therefore not seek further ethical approval to undertake these analyses.

The study will be reported in accordance with the Preferred Reporting Items for a Systematic Review and Meta-analysis of IPD statement. ${ }^{24}$ Findings will be published as open access articles in high-impact peerreviewed journals and presented at international conferences. We will seek to inform national, European and global developers of clinical guidelines, including the UK NICE guidance, National Health Service commissioners, the British and Irish Hypertension Society and local healthcare providers. We will coproduce a targeted dissemination plan for the public and specific patient groups and our funding charities, in conjunction with the project PPI advisory group. We also plan to undertake a public dissemination event for patients, clinicians and providers or commissioners regarding the importance of, and relationship between, arm and leg BPs and understanding the importance of BP measurement in cardiovascular risk estimation-the findings from this study will be presented. The INTERPRESS-IPD Collaboration is a large, international dataset with both arm and leg BPs, 
and is available for further research activity in this area in the future.

\section{DISCUSSION}

There are 1.2 million stroke survivors living in the UK (State of the Nation Stroke statistics-January 2017: The Stroke Association) and $75 \%$ of these individuals report weakness of upper limb function that interferes with activities of daily living. ${ }^{25}$ Self-monitoring and selftitration of BP lowering treatment achieves lower BPs in people at high risk of new or recurrent stroke.${ }^{26}$ However, this is either impossible or difficult for many stroke survivors with significantly impaired upper limb function, and for individuals with other barriers to BP measurement in the arm. Data suggest a prevalence of 12-13 individuals per 100000 population have upper limb prostheses in the UK and Norway. ${ }^{27} 28$ In addition, over 1700 amputations higher than wrist level occur annually in the UK. ${ }^{29}$ Congenital upper limb deformities are also important; for example, the UK Thalidomide Trust has 460 beneficiaries who are now aged in their late 50s. Hypertension is a particular concern in this cohort, and over half of beneficiaries report upper limb damage. ${ }^{30}$ Taking these data together, we conservatively estimate that between 6000 and 10000 adults may be living with significant congenital or acquired upper limb loss in the UK. As a population, these individuals are in particular need of accurate estimates of $\mathrm{BP}$ to understand and mitigate their cardiovascular risk, stroke being an important avoidable consequence.

Thus, barriers to accurate upper arm BP measurement exist for a substantial minority of the UK population, and corresponding proportions across other countries. Whenever circumstances require leg BP measurement, it is important to be able to interpret the readings correctly. This is the focus of our proposal. Our data originate from cohorts across Europe, North America and Africa; therefore, we expect our findings to be applicable across the globe.

To date, estimates suggest either a minimum difference of $15 \mathrm{~mm} \mathrm{Hg}$ in SBPs between arm and leg, or a conversion factor of $\times 0.88$, as a rule of thumb. ${ }^{511}$ This study aims to provide the first evidence-based method for estimating individual brachial SBP and cardiovascular risk from leg SBP measurements. Our findings will support clinicians and patients in detecting and managing hypertension more effectively where leg measurements are required.

\footnotetext{
Author affiliations

${ }^{1}$ Primary Care Research Group, University of Exeter, Exeter, UK

${ }^{2}$ Nuffield Department of Primary Care Health Sciences, University of Oxford, Oxford, UK

${ }^{3}$ NIHR CLAHRC South West Peninsula (PenCLAHRC), University of Exeter, Exeter, UK

${ }^{4}$ Patient and Public Involvement Advisor, Exeter, UK

${ }^{5}$ Department of Cardiology, Stockport NHS Foundation Trust, Stockport, UK

${ }^{6}$ Royal National Orthopaedic Hospital Stanmore, Stanmore, UK

${ }^{7}$ Mid Devon Medical Practice, Exeter, UK

${ }^{8}$ Institute of Clinical Sciences, University of Birmingham, Birmingham, UK
}

${ }^{9}$ Department of Cardiology, Centre Hospitalier Universitaire de Limoges, Limoges, France

Twitter James P Sheppard @jamessheppard48 and Christopher E Clark @ INTERPRESS_IPD

Collaborators The following collaborating authors contributed data to the original INTERPRESS-IPD Collaboration and the subsequent ABLE-BP project: Vietnam Experience Study: James White; INCHIANTI: Luigi Ferrucci; Heinz-Nixdorf Recall Study: Raimund Erbel; SMART: Jan Westerink; San Diego Population Study: Michael Criqui; Fuencarral Health Center: Carlos Lahoz; EPIDEMCA: Maëlenn Guerchet; MESA: Matthew Allison; LIFE \& WALCS: Mary McDermott; Look AHEAD: Mark Espeland; ViWoCo: Marie Dahl; SUMMIT: Angela Shore; MARK Study: Rafel Ramos Blanes

Contributors This protocol was first drafted by STJM, CEC, FCW, JPS, UM, LF, KB and VA, then revised and edited by all authors, including HS, PW, PSL, RB and JF. The final manuscript has been read, reviewed and approved by all authors.

Funding The INTERPRESS-IPD Collaboration was established with a grant from the NIHR Research for Patient Benefit programme (award/grant number: PB-PG-021536009). This current project is supported by the Stroke Association (award/grant number: SA PG 19/100043) and by the Thalidomide Trust (award/grant number: not applicable).

Disclaimer The views expressed are those of the authors and not necessarily those of the Stroke Association, the Thalidomide Trust, the NIHR, the NHS or the Department of Health.

Competing interests CEC has been loaned a bilateral blood pressure monitor for unrestricted evaluation by Microlife AG, and has received honoraria from Bayer AG. No company has had, or will have, any involvement in the design or conduct of this study.

Patient and public involvement Patients and/or the public were involved in the design, or conduct, or reporting, or dissemination plans of this research. Refer to the Methods section for further details.

Patient consent for publication Not required.

Provenance and peer review Not commissioned; externally peer reviewed.

Open access This is an open access article distributed in accordance with the Creative Commons Attribution Non Commercial (CC BY-NC 4.0) license, which permits others to distribute, remix, adapt, build upon this work non-commercially, and license their derivative works on different terms, provided the original work is properly cited, appropriate credit is given, any changes made indicated, and the use is non-commercial. See: http://creativecommons.org/licenses/by-nc/4.0/.

\section{ORCID iDs}

Sinead T J McDonagh http://orcid.org/0000-0002-0283-3095

James P Sheppard http://orcid.org/0000-0002-4461-8756

Kate Boddy http://orcid.org/0000-0001-9135-5488

Christopher E Clark http://orcid.org/0000-0002-7526-3038

\section{REFERENCES}

1 Whelton PK, Carey RM, Aronow WS. ACC/AHA/AAPA/ABC/ACPM/ AGS/APhA/ASH/ASPC/NMA/PCNA guideline for the prevention, detection, evaluation, and management of high blood pressure in adults: a report of the American College of Cardiology/American heart association Task force on clinical practice guidelines. J Am Coll Cardiol 2017

2 Williams B, Mancia G, Spiering W, et al. 2018 ESC/ESH guidelines for the management of arterial hypertension. Eur Heart $J$ 2018;39:3021-104.

3 National Institute for Health and Clinical Excellence. Hypertension: the clinical management of primary hypertension in adults, CG127. London: National Institute for Health and Clinical Excellence, 2011.

4 Hwang KO, Aigbe A, Ju H-H. Barriers to accurate blood pressure measurement in the medical office. J Prim Care Community Health 2018;9:215013271881692.

5 Shiga T, Shimbo T, Yoshizawa A. Multicenter investigation of lifestylerelated diseases and visceral disorders in thalidomide embryopathy at around 50 years of age. Birth Defects Res A Clin Mol Teratol 2015;103:787-93.

6 Dewar R, Sykes D, Mulkerrin E, et al. The effect of hemiplegia on blood pressure measurement in the elderly. Postgrad Med $\mathrm{J}$ 1992;68:888-91. 
7 Maduagwu SM, Umeonwuka Cl, Mohammad HH, et al. Reference arm for blood pressure measurement in stroke survivors. Middle East $J$ Rehabil Health Stud 2018;5:e62368.

8 Aboyans V, Kamineni A, Allison MA, et al. The epidemiology of subclavian stenosis and its association with markers of subclinical atherosclerosis: the multi-ethnic study of atherosclerosis (MESA). Atherosclerosis 2010;211:266-70.

9 Pascarelli EF, Bertrand CA. Comparison of blood pressures in the arms and legs. N Engl J Med 1964;270:693-8.

10 Stewart HJ, Newman AA, Evans WF. Levels of blood pressure in both arms and legs in normal subjects and patients suffering from certain diseases. Am J Med 1946;1:451-63.

11 Sheppard JP, Albasri A, Franssen M, et al. Defining the relationship between arm and leg blood pressure readings: a systematic review and meta-analysis. $J$ Hypertens 2019;37:660-70.

12 Debray TPA, Damen JAAG, Snell KIE, et al. A guide to systematic review and meta-analysis of prediction model performance. BMJ 2017;356:i6460.

13 Tudur Smith C, Marcucci M, Nolan SJ, et al. Individual participant data meta-analyses compared with meta-analyses based on aggregate data. Cochrane Database Syst Rev 2016;9:MR000007.

14 Abo-Zaid G, Sauerbrei W, Riley RD. Individual participant data metaanalysis of prognostic factor studies: state of the art? BMC Med Res Methodol 2012;12:56

15 Clark CE, Boddy K, Warren FC, et al. Associations between interarm differences in blood pressure and cardiovascular disease outcomes: protocol for an individual patient data meta-analysis and development of a prognostic algorithm. BMJ Open 2017;7:e016844.

16 Aboyans V, Criqui MH, Abraham P, et al. Measurement and interpretation of the Ankle-brachial index. Circulation2012;126:2890-909.

17 Hayden JA, van der Windt DA, Cartwright JL, et al. Assessing bias in studies of prognostic factors. Ann Intern Med 2013;158:280.

18 Altman DG, Vergouwe $\mathrm{Y}$, Royston P, et al. Prognosis and prognostic research: validating a prognostic model. BMJ 2009;338:b605.

19 Hippisley-Cox J, Coupland C, Vinogradova Y, et al. Predicting cardiovascular risk in England and Wales: prospective derivation and validation of QRISK2. BMJ 2008;336:1475-82.

20 Goff DC, Lloyd-Jones DM, Bennett G, et al. 2013 ACC/AHA guideline on the assessment of cardiovascular risk: a report of the American College of Cardiology/American heart association task force on practice guidelines. Circulation 2014:129:S49-73.

21 D'Agostino RB, Vasan RS, Pencina MJ, et al. General cardiovascular risk profile for use in primary care: the Framingham heart study. Circulation 2008:117:743-53.

22 Conroy Ret al. Estimation of ten-year risk of fatal cardiovascular disease in Europe: the score project. Eur Heart J 2003;24:987-1003.

23 Akaike $\mathrm{H}$. A new look at the statistical model identification. IEEE Trans Automat Contr 1974;19:716-23.

24 Stewart LA, Clarke M, Rovers M, et al. Preferred reporting items for a systematic review and meta-analysis of individual participant data. JAMA 2015;313:1657-65.

25 Lawrence ES, Coshall C, Dundas R, et al. Estimates of the prevalence of acute stroke impairments and disability in a multiethnic population. Stroke 2001;32:1279-84.

26 McManus RJ, Mant J, Haque MS. Effect of self-monitoring and medication Self-titration on systolic blood pressure in hypertensive patients at high risk of cardiovascular disease: the TASMIN-SR randomized clinical TrialSelf-monitoring and Self-titration for HypertensionSelf-monitoring and Self-titration for hypertension. JAMA 2014;312:799-808.
27 Kyberd PJ, Beard DJ, Morrison JD. The population of users of upper limb prostheses attending the Oxford limb fitting service. Prosthet Orthot Int 1997;21:85-91.

28 Østlie K, Skjeldal OH, Garfelt B, et al. Adult acquired major upper limb amputation in Norway: prevalence, demographic features and amputation specific features. A population-based survey. Disabil Rehabil 2011:33:1636-49.

29 Cordella F, Ciancio AL, Sacchetti R, et al. Literature review on needs of upper limb prosthesis users. Front Neurosci 2016;10:209-09.

30 Newbronner E, Glendinning C, Atkin K, et al. The health and quality of life of thalidomide survivors as they age - evidence from a UK survey. PLoS One 2019;14:e0210222.

31 McDermott MM, Greenland P, Liu K, et al. Leg symptoms in peripheral arterial disease: associated clinical characteristics and functional impairment. JAMA 2001;286:1599-606.

32 Guerchet M, Mbelesso P, Ndamba-Bandzouzi B, et al. Epidemiology of dementia in central Africa (EPIDEMCA): protocol for a multicentre population-based study in rural and urban areas of the central African Republic and the Republic of Congo. Springerplus 2014:3:338

33 Lahoz C, Barrionuevo M, Garcia-Fernandez T. Cardiovascular morbidity-mortality associated to ankle-brachial index in the general population. [Spanish]. Revista Clinica Espanola 2014;214:1-7.

34 Erbel R, Möhlenkamp S, Moebus S, et al. Coronary risk stratification, discrimination, and reclassification improvement based on quantification of subclinical coronary atherosclerosis: the heinz Nixdorf recall study. J Am Coll Cardiol 2010;56:1397-406.

35 Clark CE, Thomas D, Llewellyn D. Inter-arm blood pressure and risk of cognitive decline in the elderly. Br J Gen Pract 2020.

36 Espeland MA, Newman AB, Sink K, et al. Associations between Ankle-brachial index and cognitive function: results from the lifestyle interventions and independence for elders trial. J Am Med Dir Assoc 2015;16:682-9.

37 Martí R, Parramon D, García-Ortiz L, Garcia-Regalado N, Garcia-Gil $\mathrm{M}$, et al. Improving intermediate risk management. mark study. $B M C$ Cardiovasc Disord 2011;11:61.

38 Espeland MA, Beavers KM, Gibbs BB, et al. Ankle-brachial index and inter-artery blood pressure differences as predictors of cognitive function in overweight and obese older adults with diabetes: results from the action for health in diabetes movement and memory study. Int J Geriatr Psychiatry 2015;30:999-1007.

39 Bild DEet al. Multi-ethnic study of atherosclerosis: objectives and design. Am J Epidemiol 2002;156:871-81.

40 Wassel CL, Loomba R, Ix JH, et al. Family history of peripheral artery disease is associated with prevalence and severity of peripheral artery disease: the San Diego population study. J Am Coll Cardiol 2011:58:1386-92.

41 Kranenburg G, Spiering W, de Jong PA, et al. Inter-arm systolic blood pressure differences, relations with future vascular events and mortality in patients with and without manifest vascular disease. Int $J$ Cardiol 2017;244:271-6.

42 Clark CE, Casanova F, Gooding K. Inter-arm blood pressure difference and arterial stiffness. J Hyperten 2014;32:e30.

43 Dahl M, Frost L, Søgaard R, et al. A population-based screening study for cardiovascular diseases and diabetes in Danish postmenopausal women: acceptability and prevalence. BMC Cardiovasc Disord 2018;18:20.

44 White J, Mortensen LH, Kivimäki M, et al. Interarm differences in systolic blood pressure and mortality among US army veterans: aetiological associations and risk prediction in the Vietnam experience study. Eur J Prev Cardiol 2014;21:1394-400. 